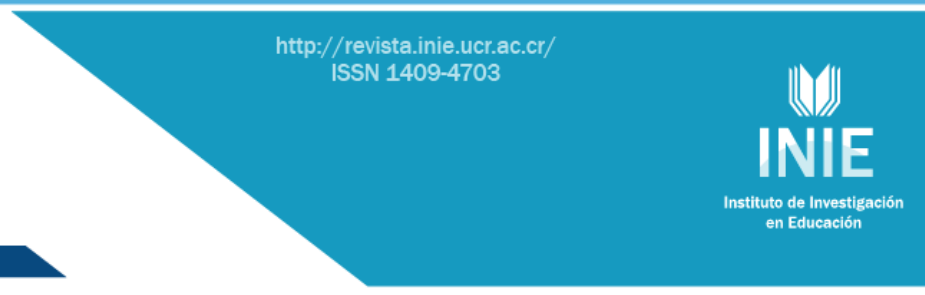

\title{
APRENDIZAJE COOPERATIVO EN UN CURSO DE BIOQUÍMICA: OPINIÓN DE ESTUDIANTES Y EFECTO EN SU RENDIMIENTO ACADEMICO
}

COOPERATIVE LEARNING IN A BIOCHEMISTRY COURSE: STUDENTS OPINION AND THE EFFECT IN THEIR ACADEMIC PERFORMANCE

\author{
Volumen 12, Número 1 \\ Enero-Abril \\ pp. 1-26
}

Este número se publicó el 28 de febrero de 2012

\author{
María Laura Fernández Montes de Oca \\ Alberto Alape-Girón \\ María José Artolozaga Sustacha \\ Luis Adolfo Calvo Bolaños \\ Carolina Centeno Cerdas \\ Georgina Gómez Salas \\ Karol Granados Blanco \\ Marvin Madrigal Villalobos \\ Ana Gabriela Murillo Solís \\ Adrián Pinto-Tomás \\ Silvia Quesada Mora \\ Elvira Salas Hidalgo \\ Luis Felipe Somarribas Patterson \\ Luisa Alejandra Vindas Angulo \\ Diana Campos Rodríguez
}

Revista indizada en REDALYC

Revista distribuida en las bases de datos:

CATÁLOGO DE LATINDEX, IRESIE, CLASE, DIALNET, DOAJ, E-REVIST@S,

Revista registrada en los directorios:

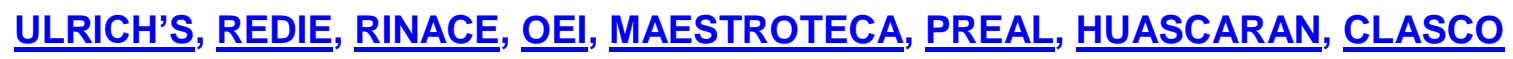




\title{
APRENDIZAJE COOPERATIVO EN UN CURSO DE BIOQUÍMICA: OPINIÓN DE ESTUDIANTES Y EFECTO EN SU RENDIMIENTO ACADEMICO
}

COOPERATIVE LEARNING IN A BIOCHEMISTRY COURSE: STUDENTS OPINION AND THE EFFECT IN THEIR ACADEMIC PERFORMANCE

\author{
María Laura Fernández Montes de Oca ${ }^{1}$ \\ Alberto Alape-Girón ${ }^{2}$ \\ María José Artolozaga Sustacha ${ }^{3}$ \\ Luis Adolfo Calvo Bolaños ${ }^{4}$ \\ Carolina Centeno Cerdas ${ }^{5}$ \\ Georgina Gómez Salas ${ }^{6}$ \\ Karol Granados Blanco ${ }^{7}$ \\ Marvin Madrigal Villalobos ${ }^{8}$ \\ Ana Gabriela Murillo Solís ${ }^{9}$ \\ Adrián Pinto-Tomás ${ }^{10}$ \\ Silvia Quesada Mora ${ }^{11}$ \\ Elvira Salas Hidalgo ${ }^{12}$ \\ Luis Felipe Somarribas Patterson ${ }^{13}$ \\ Luisa Alejandra Vindas Angulo ${ }^{14}$ \\ Diana Campos Rodríguez ${ }^{15}$ \\ Universidad de Costa Rica
}

\begin{abstract}
Resumen: El curso MQ-203 Bioquímica para Microbiología, fue impartido del 2006 al 2010 mediante clases magistrales en donde la evaluación del aprendizaje de los estudiantes se llevó a cabo exclusivamente mediante exámenes de selección única. En el primer semestre del 2011, además de las clases magistrales, en este cuso se implementaron varias actividades de aprendizaje cooperativo incluyendo trabajos en grupo, el estudio y la discusión de casos clínicos y el desarrollo de un trabajo de investigación en grupo. Estas actividades tuvieron un valor de $25 \%$ en la nota final del curso. En este artículo se analizan las opiniones de los estudiantes sobre estos cambios y el efecto de estos en su desempeño. Los estudiantes expresaron sentir un mayor compromiso con el proceso de aprendizaje, tanto individual como el de sus compañeros y mostraron una gran satisfacción con respecto al desarrollo del curso. Además, los estudiantes obtuvieron mejores notas que en años anteriores y aumentó significativamente el porcentaje de aprobación del curso. De esta manera, la implementación de actividades cooperativas contribuyó a una mejora en la disposición de los estudiantes hacia el curso y a su desempeño en el mismo.
\end{abstract}

Palabras clave: APRENDIZAJE, BIOQUÍMICA, MICROBIOLOGÍA, APRENDIZAJE COOPERATIVO, DESEMPEÑO.

Abstract: The course MQ-203 Biochemistry for microbiology students was taught between 2006 and 2010 through lectures in which the learning process of the students was assessed exclusively by single-selection tests. During the first term of the year 2011, besides lectures, several cooperative learning activities were implemented in this course, such as assignments in groups, the study and discussion of clinical cases, and the development of a research project in groups. These activities had a weight of $25 \%$ in the final grade of the course. In this paper student's opinion about these changes are analyzed as well as their effect on their performance. Students reported a greater commitment to, both, personal and classmate's learning process, and exhibit high satisfaction with the development of the course. Furthermore, there was a significant increase in the mean score of the students' compared to previous years, as well as in the rate of students who approved the course. Thus, the implementation of cooperative activities contributed to a better attitude toward the curse and significantly improved their performance.

Keywords: LEARNING, BIOCHEMISTRY, MICROBIOLOGY, COOPERATIVE LEARNING, PERFORMANCE. 


\section{Introducción}

La bioquímica es la ciencia que estudia la base química de la vida, los componentes químicos de las células, las reacciones en las que participan y su regulación (Murray, 2003). Los conocimientos de los microbiólogos en esta disciplina son de gran importancia para el ejercicio de la profesión ya que son indispensables para la comprensión de los procesos metabólicos básicos de microorganismos, plantas, animales y humanos (Prescott et al., 2002).

El curso MQ-203 Bioquímica para Microbiología de la Universidad de Costa Rica, es un curso teórico de seis créditos que se imparte en el primer semestre del tercer año de la carrera de microbiología (quinto ciclo), cuando los estudiantes han aprobado los cursos de Biología General, Química General, Química Analítica, Química Orgánica, Anatomía Macroscópica e Histología. El curso supone el aprendizaje de una gran cantidad de información, tiene una intensidad de tres sesiones de dos horas por semana y tradicionalmente se ha impartido mediante clases magistrales impartidas por el profesor. Del 2006 al 2010 el curso MQ-203 Bioquímica para Microbiología, contó usualmente con la participación de cuatro profesores por semestre, y la evaluación estuvo basada exclusivamente en exámenes escritos de selección única, con cuatro a seis opciones por pregunta.

En los años 2006 y 2007 se realizaron cuatro exámenes parciales no acumulativos de $25 \%$ cada uno, en el año 2008 cinco exámenes parciales no acumulativos de $20 \%$ cada uno, en el año 2009 tres parciales no acumulativos de $20 \%$ cada uno y un examen final acumulativo del $40 \%$, y en el año 2010 cuatro parciales no acumulativos de $15 \%$ cada uno y un examen final acumulativo del $40 \%$. Estas variaciones en la cantidad de exámenes se realizaron para tratar de mejorar el rendimiento de los estudiantes que obtenían notas promedio de 6.3 a 6.9 , y los porcentajes de aprobación del curso los cuales estaban entre el $54 \%$ y el $78 \%$.

Debido a la baja promoción del curso MQ-203 Bioquímica para Microbiología, la cátedra de bioquímica decidió realizar un cambio sustantivo en este curso al plantear el cambio del modelo de enseñanza como parte de la solución a este problema. Se decidió implementar diversas actividades de aprendizaje cooperativo para incentivar al alumno, y formarlo en temas relacionados con la bioquímica, así como para diversificar la evaluación del desempeño de los estudiantes. El propósito de esta investigación fue identificar el 
impacto de la incorporación de actividades de aprendizaje cooperativo en el rendimiento de los estudiantes y el aprovechamiento en el curso MQ-203 Bioquímica para Microbiología, así como la respuesta de los estudiantes ante los cambios implementados.

Los primeros catorce autores de este artículo participaron en el planeamiento de los cambios a realizar en la metodología de enseñanza en el curso en cuestión, el planeamiento de las clases, las actividades en grupo, la revisión de trabajos y demás actividades propias de los docentes. Todos los autores participaron en la recolección de los datos. Los dos primeros autores realizaron el análisis de los datos y la redacción del artículo, debido a la distribución de carga académica durante el semestre posterior al curso. Todos los autores revisaron el artículo e hicieron sugerencias para mejorarlo.

\section{Referente teórico}

\subsection{El aprendizaje cooperativo}

El aprendizaje cooperativo es un modelo pedagógico basado en el trabajo en grupo para la construcción colectiva del conocimiento. Este modelo está fundamentado en el enfoque pedagógico socio histórico de Vigotsky, que destaca la importancia de las interacciones sociales, lenguaje y cultura en el proceso de aprendizaje (Chew et al., 2008; Smagorinsky, 2007). En las actividades de aprendizaje cooperativo los estudiantes trabajan en grupos heterogéneos, permanentes y estructurados de tres a cinco estudiantes en los cuales cada miembro tiene responsabilidades definidas y es co-responsable del aprendizaje de los otros miembros del grupo. Los grupos evalúan permanentemente su desempeño y la calidad de su trabajo para buscar la mejora continua. Las actividades de aprendizaje cooperativo permiten a los estudiantes entender los conceptos a ser aprendidos al producir conflictos cognitivos entre individuos con opiniones divergentes que deben intercambiar ideas para llegar a un consenso (Baumberger-Henry, 2005; Domingo, 2008). El aprendizaje cooperativo es subutilizado ya que muchos estudiantes no entienden la forma de trabajar cooperativamente con otros, debido a que la cultura que prevalece en nuestras sociedades y universidades, está orientada hacia el trabajo competitivo e individualista (Johnson et al., 1998).

Sin embargo, algunos estudios han demostrado que el uso de estrategias de aprendizaje cooperativo mejora la percepción de los estudiantes en la resolución de problemas, en la capacidad de tomar decisiones, en sus habilidades de comunicación, 
promueve el sentido de responsabilidad social, y tiene un impacto significativamente positivo en el rendimiento de los estudiantes debido al mejoramiento de los resultados académicos (Anderson et al, 2005; Baghcheghi et al., 2011; Hijzen et al., 2007).

En este sentido, el aprendizaje cooperativo permite a los estudiantes desarrollar competencias relacionadas con la comunicación y habilidades sociales (integración, respeto, tolerancia, solidaridad) que les son útiles para relacionarse con otros (Baumberger-Henry, 2005; Domingo, 2008). En el modelo de aprendizaje cooperativo, se requiere no sólo de un mayor esfuerzo por parte de los estudiantes, sino también de los profesores (Sand-Jecklin, 2007). El papel del docente es enseñar a los estudiantes a cooperar de forma positiva y ayudarlos a resolver los problemas que puedan surgir (Baumberger-Henry, 2005; Domingo, 2008).

\section{Metodología}

\subsection{Introducción del curso MQ-203 Bioquímica para Microbiología del año 2011 a los estudiantes}

La carga académica destinada en años anteriores al curso MQ-203 Bioquímica para Microbiología fue de $3 / 4$ de tiempo completo, mientras que este semestre, se aumentó a 2 1/2 tiempos completos. Esto permitió la participación de 20 profesores de diferentes disciplinas. En cada sesión de trabajo participaron de dos a tres profesores, de manera que además de la clase magistral, se procuró realizar una actividad en grupos o asignar una tarea en grupo extraclase. Con respecto a la evaluación del aprendizaje de los estudiantes, el $75 \%$ de la nota incluyó cinco exámenes parciales escritos no acumulativos y 9 pruebas cortas individuales, mientras que el 25\% restante incluyó la evaluación de las actividades de aprendizaje cooperativo.

Con el fin de motivar la participación de los estudiantes en el curso, durante la primera semana de clase se realizaron actividades donde los estudiantes investigaron acerca de la importancia del curso para el ejercicio de la profesión. Se realizó una serie de charlas cortas relacionadas con la microbiología, la bioquímica y el aprendizaje con los siguientes títulos: "El perfil del microbiólogo y químico clínico que se quiere formar en la Universidad de Costa Rica”, “Importancia de la bioquímica en el ejercicio de la microbiología”, “¿Cómo aprende el cerebro humano?", "Evolución de la vida sobre la tierra", 'Microorganismos extremófilos" y "Vida extraterrestre". 
A los estudiantes se les presentó el Aula Virtual del curso, el cual es un espacio en la red, utilizado para el aprendizaje a distancia para facilitar la interacción por medio de aplicaciones como foros, encuestas, formularios y acceso a documentación relacionada con el curso (http://www.fmedicinaenlinea.ucr.ac.cr/moodle/). Este espacio fue utilizado para que los estudiantes tuvieran acceso a las presentaciones de las clases, capítulos de libros de consulta, artículos científicos, asignaciones de trabajos grupales, entrega virtual de trabajos, pruebas cortas individuales en línea y participación en foros de discusión. Además, se habilitó un foro permanente a través del semestre, para que los estudiantes tuvieran la oportunidad de expresar sus opiniones sobre las actividades del curso. De manera que, este espacio fue de gran utilidad porque permitió a los estudiantes tener acceso inmediato y permanente al material del curso, e interactuar con los docentes a través de los foros. Además, como parte de las modificaciones implementadas en el año 2011, se realizó la definición conjunta de objetivos generales del curso entre profesores y estudiantes, los cuales fueron discutidos en clase e incorporados a la versión final del programa del curso.

\subsection{Descripción de actividades para el aprendizaje cooperativo en el curso MQ-203 Bioquímica para Microbiología del año 2011}

La conformación de los grupos de trabajo se hizo al azar, utilizando como guía la lista de clase de estudiantes matriculados. Se formaron trece grupos: nueve grupos con cuatro estudiantes y tres grupos con tres estudiantes, incluyendo a un repitente por equipo de trabajo. Las actividades en grupo incluyeron: actividades en clase, tareas grupales, estudio y discusión de casos clínicos y un trabajo de investigación.

Como trabajo en clase se consideraron todas aquellas actividades participativas, al inicio o al final de la clase, en donde por medio de preguntas, crucigramas o juegos, los estudiantes mostraran sus conocimientos. Las tareas y trabajos grupales fueron todas aquellas asignaciones que los estudiantes debían preparar en horas extra clase y presentar en una fecha determinada. Algunas de estas tareas fueron asignadas y entregadas mediante el aula virtual.

Durante el desarrollo del curso, se realizaron tres sesiones de análisis bioquímico de casos clínicos, los cuales debían ser discutidos por el grupo de estudiantes antes de la sesión. Los profesores elaboraron una lista de preguntas sobre cada caso y se asignó una pregunta a cada grupo, la cual debía ser resuelta y presentada al resto de estudiantes en 
una fecha establecida. Mediante el estudio de casos clínicos, se le presenta a los estudiantes una historia atractiva o controversial, para la cual, requerían de un entendimiento básico de principios científicos. De esta manera, los estudiantes comprenden conceptos, retienen el contenido, y logran un pensamiento crítico al desarrollar capacidades para resolver problemas de la vida real (Rybarczyk et al., 2007). Los casos clínicos a discutir, tomados del libro Clinical Studies in Medical Biochemistry, fueron: "Anemia falciforme" (Quirolo 2007), “Deficiencia de a-1 antitripsina” (Schwarzenberg et al.,2007) y "Malnutrición proteico-energética" (Gopichandran et al.,2007).

Para el trabajo de investigación, cada grupo escogió un tema a partir de una lista propuesta por los profesores del curso. Cada grupo debía de desarrollar el tema bajo la tutoría de un profesor, incluyendo la elaboración de un anteproyecto, un trabajo escrito final y una presentación oral al resto del grupo y profesores. Este trabajo de investigación sigue el modelo del aprendizaje basado en proyectos, en donde los estudiantes deben expresar habilidades como trabajo en equipo, manejo de conflicto, toma de decisiones, y habilidades de comunicación. Mediante estas investigaciones, los estudiantes no sólo aprenden, sino también fortalecen habilidades relacionadas con la seguridad, el pensamiento crítico, la generación y discusión de ideas y productividad (De los Ríos et al., 2010; Musa et al., 2011).

Los temas de los trabajos fueron los siguientes: 1. Papel del estrés oxidativo en el daño tisular en infarto cerebral, 2. Neurotransmisores y desórdenes mentales, 3. Marcadores de disfunción endotelial, 4. Quórum sensing y patogénesis, 5. Muerte celular y envejecimiento, 6. Priones como agentes de enfermedades en humanos, 7. Nutrigenética y nutrigenómica, 8. Índice glicémico y enfermedades crónicas, 9. Papel de los microARN's en la regulación de la expresión génica y posibles aplicaciones terapéuticas, 10. Fenilcetonuria y galactosemia, 11. Acciones cerebrales de los esteroides, 12. Células Madre tumorales, 13. Factores nutricionales en diabetes.

Como se mencionó anteriormente, el $25 \%$ de la nota de los estudiantes correspondía a la evaluación de actividades cooperativas. Dentro de este rubro se le asignó un valor del $10 \%$ a las actividades y asignaciones en clase y extra clase, así como el desarrollo de los casos clínicos, y un 15\% al trabajo de investigación. Además, al entregar un trabajo en grupo los estudiantes debían indicar al profesor una nota que reflejara el aporte de cada uno de los participantes, la cual se multiplicaría por la nota asignada por el profesor. Así cada estudiante tendría una nota diferenciada según su contribución a cada trabajo. 


\subsection{Caracterización del perfil socioeconómico de los estudiantes}

Para conocer las características socioeconómicas de los estudiantes que matricularon el curso MQ-203 Bioquímica para Microbiología durante el primer semestre del 2011, al inicio del curso se les solicitó contestar un cuestionario elaborado por los profesores del curso (Cuadro No.1).

\section{CUADRO No.1.}

Cuestionario: Información del estudiante

\section{Perfil socioeconómico del estudiante:}

1. Edad: 17-19 años / 20-23 años / $\geq 24$

2. Sexo: Masculino / Femenino

3. Estado civil: Soltera(o) / Casada(o)

4. ¿Tiene hijos?: No / Si

5. La mayor parte de su vida ha residido en: Zona Rural / Zona Urbana

6. ¿Actualmente Trabaja?: No / Sí.

7. Marque los bienes a los cuales tiene acceso: Internet / Computadora personal / Vehículo / Celular / Cable / Tarjeta de crédito

8. ¿Se encuentra becado? Sin beca / Beca 1-9 / Beca 10 / Beca 11

\section{Perfil educativo}

1. ¿Dónde realizó sus estudios secundarios?: Colegio Privado / Colegio Público / Colegio Científico / Colegio Técnico-Profesional

2. Año de ingreso a la carrera: 2009 / 2008 / antes del 2008

3. ¿Fue Microbiología su primera opción a la hora de escoger carrera?: Si / No

4. ¿Lleva el curso por primera vez?: No / Si

5. ¿Lleva bloque completo de estudios?: No / Si

\section{Información de los padres}

1. Situación actual: Casados / Divorciados / Viuda(o) / Unión libre

2. Nivel educativo de los padres: Primaria / Secundaria / Estudios Universitarios

3. ¿Cuenta con apoyo económico de padres o algún familiar para sus estudios?: No / Si

\subsection{Examen diagnóstico de conocimientos básicos}

Con el fin de conocer el nivel de conocimientos básicos de los estudiantes con respecto a las materias previamente aprobadas, las cuales son requisito para poder llevar el curso de bioquímica, se llevó a cabo un examen diagnóstico. Esta evaluación se realizó durante la semana de introducción al curso, con un tiempo asignado de desarrollo de 35 minutos. Esta prueba contenía 15 preguntas de selección única con 5 opciones por pregunta, de las cuales 5 preguntas eran de Biología General, 4 preguntas de Química 
General, 4 preguntas de Química Orgánica y 2 preguntas de Histología. Los resultados de esta prueba fueron presentados en clase luego de su revisión.

\subsection{Opiniones iniciales de los estudiantes frente a la introducción de las actividades de aprendizaje cooperativo}

Durante la segunda semana de clases se plantearon las siguientes preguntas en el aula virtual, para conocer la opinión de cada estudiante sobre la introducción de actividades de aprendizaje cooperativo y parámetros de evaluación del aprendizaje: ¿Qué expectativas le genera la metodología que se va a usar en este curso y los parámetros de evaluación del aprendizaje?, ¿Qué espera usted de los compañeros y las compañeras de su grupo de trabajo? y ¿A qué se compromete usted con los y las compañeros(as) de su grupo de trabajo?. Además se les consultó que, según una escala del 1 al 5 ("1"= desmotivado y "5" = muy motivado) indicaran el grado de motivación con respecto al curso.

Durante la tercera semana del curso, se les solicitó a los estudiantes que, de forma individual, respondieran las siguientes preguntas sobre el funcionamiento de los grupos hasta ese momento: ¿Se definieron las funciones de los miembros del grupo y cada cuánto rotarán las funciones?, ¿Cuáles son las principales fortalezas y debilidades de su grupo?, ¿Han tenido que tomar decisiones como grupo y tuvieron problemas tomando alguna de estas? y ¿Considera que ha habido conflictos de liderazgo en su grupo?

\subsection{Correlación entre las variables socioeconómicas y asistencia a sesiones de repaso, con el rendimiento individual de los estudiantes}

Con el fin de determinar si hay alguna o varias variables que afecten significativamente el rendimiento académico individual de los estudiantes en el curso, se realizó un análisis de correlación entre las variables indicadas en cuadro 1, y la nota obtenida por cada estudiante de ser evaluado únicamente el $75 \%$ de rendimiento individual que incluía exámenes parciales y pruebas cortas (nota individual). Además, durante el curso, previo a cada examen, se realizó una sesión de repaso para un total de 5 sesiones, para despejar dudas, aclarar conceptos y realizar una práctica de los temas a evaluar en el examen. Se registró la asistencia de los estudiantes a estas sesiones y se realizó un análisis de correlación entre la asistencia y la nota individual obtenida por cada estudiante. 


\subsection{Evaluación del efecto de la introducción de actividades de aprendizaje cooperativo en el aprovechamiento y rendimiento de los estudiantes}

Con el objetivo de evaluar el efecto de la introducción de estrategias de aprendizaje cooperativo en el desempeño de los estudiantes en el curso MQ-203 Bioquímica para Microbiología, se realizaron análisis comparativos de los porcentajes de aprovechamiento y rendimiento obtenidos por los estudiantes en los cursos de los años 2006 al 2010 versus el curso del 2011. Además, se comparó la nota final obtenida en el curso con la nota individual de haber sido evaluado únicamente el $75 \%$ de rendimiento individual.

\subsection{Evaluación del impacto de la introducción de actividades cooperativas en el aprendizaje de los estudiantes}

Para evaluar el impacto de la introducción de estrategias de aprendizaje cooperativo en el aprendizaje de los estudiantes del curso MQ-203 Bioquímica para Microbiología al finalizar el semestre, se les solicitó llenar un cuestionario final en el aula virtual, donde expresaron su opinión sobre la implementación de estrategias de aprendizaje cooperativo en el curso, y qué tanto les ayudó estas al proceso de aprendizaje (ver preguntas en Cuadro No.3)

\section{Resultados y discusión}

\subsection{Características socioeconómicas de los estudiantes}

Se matricularon 49 estudiantes en el curso, de los cuales el $21 \%$ eran repitentes y el $79 \%$ lo cursaban por primera vez. El 53\% de los estudiantes matriculó el curso de bioquímica junto con el resto de cursos establecidos en el quinto bloque de la carrera. El $60 \%$ de los estudiantes tenía una edad entre los 20 y 23 años, un $22 \%$ tenía 24 años o más, y el $18 \%$ restante tenía entre 17 y 19 años. La mayoría del grupo contaba con las siguientes características: mujeres $(71.5 \%)$, solteros o solteras $(92 \%)$, sin hijos $(90 \%)$, y sin trabajo (82\%). Del $18 \%$ de estudiantes que mencionaron trabajar, el $10 \%$ lo hace a tiempo parcial y el $8 \%$ a tiempo completo. El $70 \%$ de los estudiantes eran becados por la universidad, con becas de 1 a $9(34 \%)$, beca $10(16 \%)$ y beca $11(20 \%)$. De manera que en general, el curso MQ-203 Bioquímica para Microbiología, fue matriculado por una población de estudiantes en su mayoría jóvenes, que entraron a la universidad al terminar sus estudios secundarios, solteros, dedicados únicamente al estudio y con algún tipo de beca. 
Al consultar acerca de los bienes a los cuales tienen acceso, contestaron: celular el $100 \%$, internet el $94 \%$, computadora personal el $80 \%$, servicio de cable el $61 \%$, tarjeta de crédito el $18 \%$ y vehículo el $16 \%$. Por lo tanto, los estudiantes en general, cuentan con un nivel socioeconómico medio. Un $57 \%$ de los estudiantes ha vivido la mayor parte de su vida en zona urbana y un $43 \%$ en zona rural. El $40 \%$ de los estudiantes vienen de colegios públicos, mientras que un $29 \%$ de colegio privado, un $25 \%$ de colegio científico y el $6 \%$ restante de colegio Técnico-Profesional. El 49\% de los estudiantes ingresó a la universidad en el año 2009, por lo que el 2011 sería su tercer año de carrera. El 29\% ingresó en el 2008, y el resto de estudiantes ingresó antes del 2008, los cuáles serían estudiantes que van rezagados uno, dos o más años en la carrera. Sorprendentemente, únicamente el $22 \%$ de los estudiantes había escogido microbiología como primera opción de carrera.

En cuanto al perfil de los padres de los estudiantes, el 69\% de los estudiantes indicó que sus padres se encuentran casados, el $27 \%$ divorciados, el $2 \%$ tiene solo uno de sus padres y el $2 \%$ restante vive en unión libre. En cuanto al nivel educativo, el $57 \%$ de los estudiantes mencionó que sus padres tienen educación universitaria, el 25\% secundaria, y el $18 \%$ únicamente primaria. El $85 \%$ de los estudiantes cuenta con apoyo económico de sus padres o algún familiar para la realización de sus estudios. Por lo tanto, la mayoría de estudiantes vienen de familias donde sus padres están casados, con estudios universitarios y que les brindan apoyo económico, lo cual indica que cuentan con un ambiente favorable para dedicarse a sus estudios.

\subsection{Resultados del examen diagnóstico}

Los resultados del examen diagnóstico muestran que únicamente el $19 \%$ de los estudiantes obtuvo una nota mayor o igual a 70 , siendo el promedio de $56.6 \pm 15.1$. Los porcentajes de aprobación de las preguntas, por tema, fueron los siguientes: Biología General 57.4\%, Química General 46\%, Química Orgánica 54\% e Histología 80.5\%. Por lo tanto, el examen diagnóstico reveló que los estudiantes tenían deficiencias en conceptos básicos de las áreas de biología general, química general y química orgánica, por lo que, al revisar el examen diagnóstico, se les indicó la importancia de repasar estos temas. Algunos estudios han relacionado la memoria de lo aprendido en clase por los estudiantes, con la forma en que lo aprenden (Nuthall, 2000). Estos resultados demuestran que la forma en que 
estos estudiantes aprendieron estos conceptos, no contribuyó a que fueran retenidos por mucho tiempo.

\subsection{Opiniones iniciales de los estudiantes acerca de la introducción de actividades de aprendizaje cooperativo}

Luego de la revisión de los objetivos generales del curso planteados por profesores y estudiantes, se aceptaron aquellos que fueron considerados adecuados para el curso y la nueva metodología. La lista final de objetivos fue más amplia que la de años anteriores.

Como se detalla en el Cuadro No.2, se incluyeron objetivos cognitivos los cuáles abarcaron no sólo el dominio de la bioquímica sino también la adquisición o mejora de habilidades científicas básicas, incluyendo análisis de datos, formulación de preguntas, y aplicación del conocimiento a situaciones complejas.

Además, debido a la metodología de aprendizaje cooperativo, se plantearon objetivos para lograr un aprendizaje social en los estudiantes, que fueran aplicables no solo en el desarrollo del curso, sino en sus vidas personales. También se incluyeron objetivos personales de superación individual. El involucrarlos en la formulación de los objetivos del curso resultó muy novedoso y motivante para ellos y se reflejó en la amplia lista de objetivos planteados, incluyendo objetivos de mejora de las habilidades científicas básicas, aprendizaje afectivo y social. 


\section{CUADRO No.2}

Objetivos generales del curso MQ0203-Bioquímica para Microbiología, I semestre 2011

\begin{tabular}{|c|c|c|}
\hline Cognitivos & Sociales & Personales \\
\hline $\begin{array}{l}\text { Aumentar la capacidad de pensar } \\
\text { de manera creativa, crítica y } \\
\text { autocrítica }\end{array}$ & $\begin{array}{l}\text { Fortalecer capacidades para } \\
\text { trabajar de manera armónica y } \\
\text { eficaz en grupos que evalúen } \\
\text { permanentemente su } \\
\text { desempeño y la calidad de su } \\
\text { trabajo }\end{array}$ & $\begin{array}{l}\text { Fomentar el desarrollo de } \\
\text { una cultura de búsqueda de } \\
\text { mejora continua }\end{array}$ \\
\hline $\begin{array}{l}\text { Mejorar la capacidad de analizar, } \\
\text { sintetizar, relacionar e integrar } \\
\text { información }\end{array}$ & $\begin{array}{l}\text { Fortalecer la capacidad para } \\
\text { entender las perspectivas de } \\
\text { los otros }\end{array}$ & $\begin{array}{l}\text { Mejorar la capacidad de } \\
\text { aprender en forma autónoma }\end{array}$ \\
\hline $\begin{array}{l}\text { Fortalecer una actitud científica } \\
\text { que motive el interés por } \\
\text { investigar de manera sistemática } \\
\text { las causas a los problemas que se } \\
\text { le presenten }\end{array}$ & $\begin{array}{l}\text { Fortalecer habilidades para } \\
\text { debatir respetando las } \\
\text { opiniones ajenas y, buscando el } \\
\text { consenso a través de la } \\
\text { argumentación }\end{array}$ & $\begin{array}{l}\text { Reconocer la importancia y } \\
\text { aplicación de la bioquímica } \\
\text { para el ejercicio profesional } \\
\text { de la Microbiología y la } \\
\text { Química Clínica }\end{array}$ \\
\hline $\begin{array}{l}\text { Mejorar la habilidad para } \\
\text { comunicarse en forma clara y } \\
\text { fluida de manera oral y escrita }\end{array}$ & $\begin{array}{l}\text { Fortalecer habilidades para } \\
\text { integrarse socialmente como el } \\
\text { respeto, la tolerancia y la } \\
\text { solidaridad }\end{array}$ & $\begin{array}{l}\text { Estimular el deseo de } \\
\text { superación constante y el } \\
\text { espíritu de sana competencia }\end{array}$ \\
\hline $\begin{array}{l}\text { Reconocer la importancia de la } \\
\text { lectura de publicaciones } \\
\text { científicas como herramienta en el } \\
\text { estudio de fenómenos asociados } \\
\text { con el ejercicio de su profesión }\end{array}$ & & \\
\hline $\begin{array}{l}\text { Adquirir interés y habilidades para } \\
\text { buscar, analizar y valorar } \\
\text { información especializada de } \\
\text { diferentes fuentes que permita la } \\
\text { actualización permanente en las } \\
\text { ciencias biomédicas. }\end{array}$ & & \\
\hline $\begin{array}{l}\text { Aprender a escribir un artículo } \\
\text { científico de revisión }\end{array}$ & & \\
\hline $\begin{array}{l}\text { Razonar críticamente frente a una } \\
\text { historia clínica identificando y } \\
\text { jerarquizando los principales } \\
\text { datos del caso }\end{array}$ & & \\
\hline $\begin{array}{l}\text { Comprender los distintos } \\
\text { procesos bioquímicos que se } \\
\text { desarrollan a nivel celular, tanto } \\
\text { en organismos procariotas como } \\
\text { eucariotas }\end{array}$ & & \\
\hline
\end{tabular}

Con respecto al grado de motivación hacia el curso, el $53.1 \%$ de los estudiantes expresó sentirse muy motivado y solo el $4.1 \%$ se manifestó desmotivado. En cuanto a la 
opinión de los estudiantes sobre la implementación de actividades de aprendizaje cooperativo en el curso solicitada la segunda semana de clases, la mayoría expresó que la metodología les parecía novedosa. Algunos pocos expresaron dudas ante los cambios y comentaron que implicaba un mayor esfuerzo de parte de ellos, por lo que sería más cansado, el tiempo podría no ser suficiente para profundizar en la materia, cumplir los objetivos y finalmente ganar el curso; y que sería difícil trabajar en equipo con compañeros desconocidos. Sin embargo la mayoría se mostró muy positiva hacia los cambios.

Debido a las asignaciones en grupo, expresaron que aprenderían a trabajar en equipo junto a personas con distinto criterio, y esto les hizo sentir un mayor compromiso con el curso y con los compañeros de equipo. Resultó ser de su agrado las actividades planteadas ya que fomentaban la investigación y el análisis, Con estos ajustes, esperaban aprender más y mejor, para luego aplicar lo aprendido en los posteriores cursos de la carrera. La gran mayoría estuvo de acuerdo con el valor asignado a los parámetros de evaluación del aprendizaje, debido a que no todo el valor de la nota dependía de exámenes, sino además se tomaba en cuenta el trabajo en grupo, tanto en clase como extra clase.

Los estudiantes comentaron que esperaban de sus compañeros y compañeras de grupo de trabajo: responsabilidad, compromiso, esfuerzo, cooperación, respeto, consideración, excelencia, buena comunicación, dedicación y trabajo equitativo. De igual forma los estudiantes comentaron comprometerse con sus compañeros y compañeras de grupo, a ser participativos, responsables, serios, comprometidos, abiertos al diálogo, esforzados, trabajadores, honestos, puntuales, solidarios; a cumplir con las tareas y no evadir ni posponer las responsabilidades.

Durante la tercera semana de clases, todos los estudiantes contestaron que ya habían definido las funciones de cada uno de los miembros del equipo. Algunos pensaban rotar estas funciones y otros habían decidido dejar las funciones asignadas durante todo el semestre. Entre las fortalezas, destacó un gran compromiso hacia el resto del grupo y un interés en cumplir con las responsabilidades, así como una buena comunicación, buenas relaciones y respeto entre los integrantes. Una minoría comentó que hacían reuniones vía internet, tenían buena organización, y contaban con suficiente tiempo para reunirse.

En cuanto a las debilidades, la gran mayoría señaló la falta de tiempo o facilidad para reunirse y hacer los trabajos. Una minoría comentó que el problema del grupo era el manejo del estrés, el choque de opiniones y la mala comunicación. Un grupo mencionó una mala 
participación de un miembro particular del equipo. También algunos estudiantes comentaron que por el momento su grupo no presentaba ningún tipo de debilidad. Para esa fecha, ya habían tomado algunas decisiones como grupo y ningún estudiante comentó que eso les hubiera causado problemas. También mencionaron que no habían tenido problemas de liderazgo. De manera que, al inicio del semestre, el trabajo en grupo fue bastante positivo; en la mayoría los estudiantes lograron acoplarse a trabajar con compañeros nuevos y estaban satisfechos con los resultados obtenidos hasta el momento.

En cuanto al cuestionario sobre el desarrollo del curso aplicado durante la semana 12, los estudiantes calificaron su esfuerzo y dedicación con una nota promedio de 8.8 \pm 1.3 (mínimo 5 y máximo 10). En cuanto a la contribución de las diferentes actividades en su formación profesional y/o a su proceso de aprendizaje se obtuvieron los siguientes puntajes con la respetiva desviación estándar, nota mínima y máxima otorgada: tareas en grupo 8.0 \pm 2.0 (1-10), actividades en grupo realizadas en la clase $7.4 \pm 2.1$ (1-10), casos clínicos 8.9 \pm 1.5 (5-10), anteproyecto de investigación $8.4 \pm 1.5$ (2-10) y proyecto de investigación $8.7 \pm 1.4$ $(2-10)$.

La mayoría de los estudiantes comentó que el principal problema individual para aprovechar al máximo el curso hasta la fecha, era la falta de tiempo para llevar la materia al día y realizar todas las actividades. Varios señalaron el llevar el bloque completo de materias, el excesivo número de tareas y trabajos, y el tener otras responsabilidades personales (casa, familia, trabajo). Sin embargo, al final del curso se encontró que los estudiantes que matricularon todas las materias del bloque obtuvieron un mejor rendimiento que los que no matricularon todos los cursos (ver "Variables que afectan el rendimiento individual de los estudiantes"). Otros pocos comentaron que el estrés, los problemas de organización y los problemas en el grupo, habían sido factores que afectaban su aprovechamiento.

Por lo tanto, conforme avanzó el semestre, los estudiantes comenzaron a presentar cansancio y estrés, lo cual se evidenció con sus participaciones en el foro de opiniones en el aula virtual. Con respecto a los problemas que como grupo habían tenido para aprovechar al máximo el curso hasta ese momento, aunque la mayoría comentó que no habían tenido problemas y que estaban trabajando bien, algunos mencionaron la falta de tiempo para reunirse $u$ organizarse, el tener que dividir el trabajo dentro del equipo, y problemas de coordinación o mala comunicación. 
Con respecto a lo más importante que habían aprendido en el curso hasta ese momento, la mayoría respondió que estaban comprendiendo y aprendiendo sobre bioquímica, a trabajar en equipo y a analizar y discutir casos clínicos. Algunos comentaron que habían aprendido a organizarse y ser responsables, a realizar proyectos de investigación, colaborar con miembros del grupo, y realizar búsquedas bibliográficas. Unos pocos comentaron que habían aprendido sobre nuevas metodologías de estudio, a ser críticos y a esforzarse cada día. La mayoría comentó que estaban conformes con el desarrollo del curso y que se debía seguir adelante con el planteamiento, ya que los problemas que habían surgido se habían solucionado oportunamente. Como recomendaciones, se menciona asignar trabajos en grupo de manera más espaciada, ya que en ocasiones tenían mucho trabajo para una misma semana.

Además, debido a la cantidad de actividades grupales, consideraban que el rubro de evaluación de trabajo cooperativo debía ser mayor al 25\% establecido. Pocos comentaron también que habrían preferido escoger a los compañeros de trabajo, ya que había un integrante que no colaboraba con el trabajo, por lo que sentían que el esfuerzo no era equitativo entre los compañeros. Unos pocos también comentaron que les hubiese gustado tener más tiempo de clases magistrales por parte de los profesores.

\subsection{Variables que afectan el rendimiento individual de los estudiantes}

De haber sido la nota final del curso únicamente en base al $75 \%$ de las pruebas individuales, la nota promedio de los estudiantes habría sido de 7.4 \pm 1.4 (con una distribución normal de las notas) y se habría obtenido una aprobación del $80 \%$ de los estudiantes en el curso. Con estos datos se procedió a determinar si había alguna o varias variables socioeconómicas que afectaran negativamente el rendimiento individual de los estudiantes (Figura No.1). Se trabajó un análisis estadístico de varianza para cada variable, y con un $95 \%$ de confianza (valores $\mathrm{p}<0.05$ ), se encontraron 7 variables que causaron notas individuales significativamente inferiores, las cuales fueron determinadas mediante la prueba LSD Fisher: edad igual o mayor a 24 años (A), estado civil casado(a) (B), trabajar (C), haber entrado a la carrera hace más de tres años (antes del 2008, D), haber escogido microbiología como primera opción de carrera $(E)$, ser repitente (F) y no llevar bloque completo de estudios $(G)$. Según estos datos, los estudiantes que se ven más afectados son aquellos que por razones laborales o familiares no han llevado un ritmo continuo en los 
estudios, por lo que no llevan el bloque completo, y por lo tanto llevan más años estudiando en la universidad.
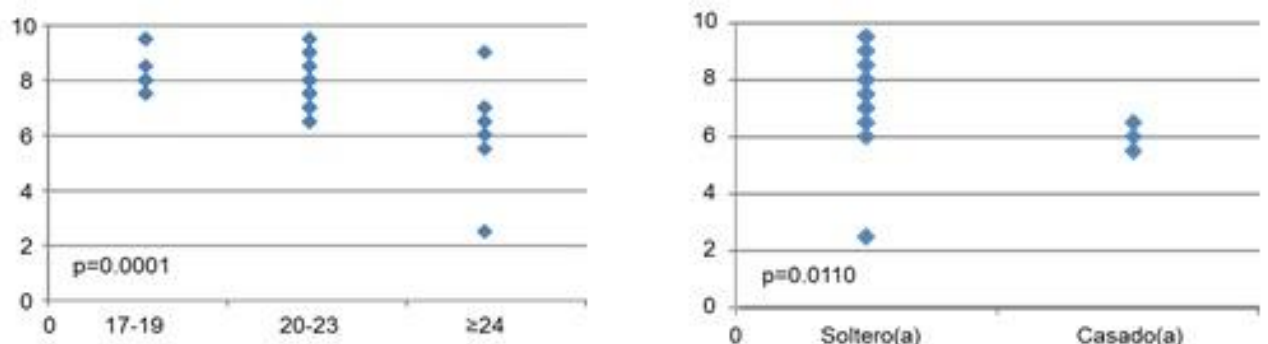

A

Edad

B

Estado civil
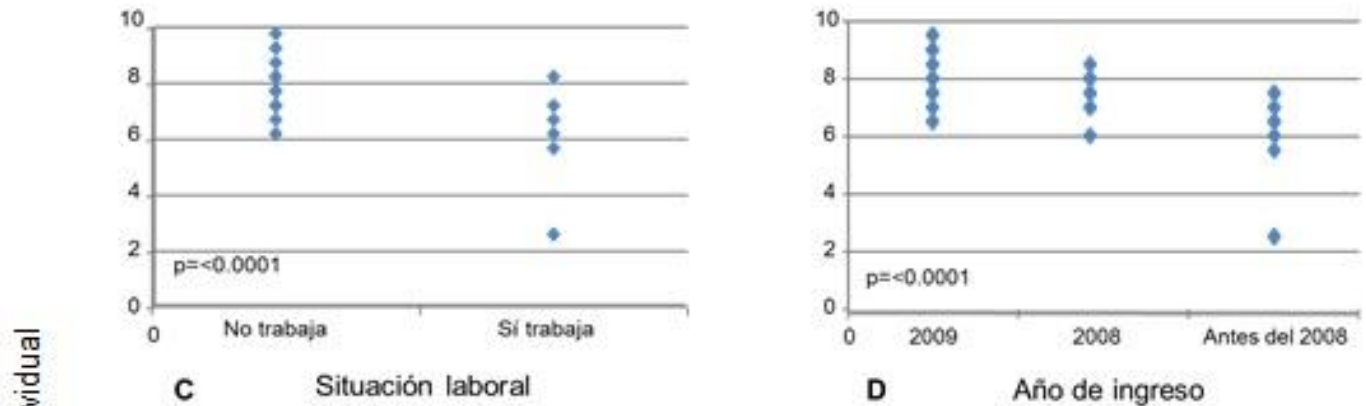

D

Año de ingreso
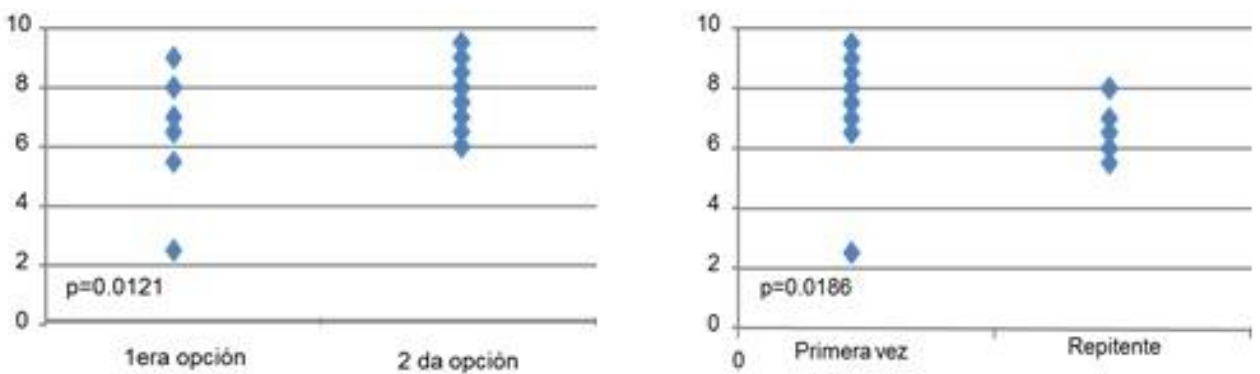

E Ingreso a microbiología

F Veces en el curso

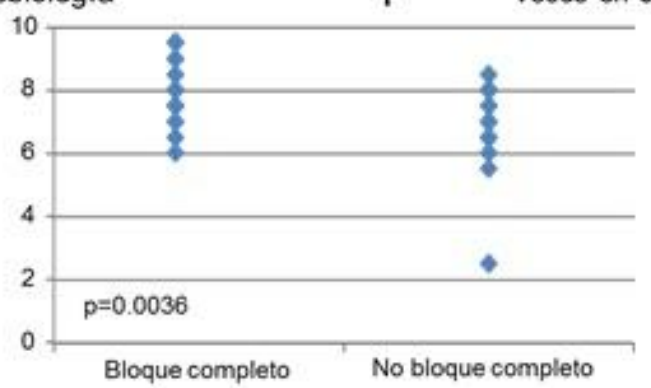

G Bloque estudios

Figura 1. Características socioeconómicas y personales, que influyen significativamente en el rendimiento de los estudiantes. 
Con respecto a las sesiones de repaso previo a los exámenes, el 14\% de los estudiantes asistió a las cinco sesiones, un $41 \%$ asistió a cuatro sesiones, un $20 \%$ a tres sesiones, un $16 \%$ a dos sesiones, un $2 \%$ a una sesión y un $6 \%$ no asistió a ninguna de las sesiones de repaso. Mediante un análisis estadístico de varianza se analizó si la asistencia a estas sesiones de repaso influyó en el desempeño individual de los estudiantes (Figura No.2). Se obtuvo un valor $p<0.05(p=<0.0001)$, con lo cual se puede afirmar, con un $95 \%$ de confianza que sí hay diferencia significativa en las notas individuales obtenidas debido a la asistencia a sesiones de repaso. Mediante la prueba LSD Fisher se determinó que aquellos estudiantes que no asistieron a ninguna sesión de repaso, obtuvieron notas significativamente inferiores a aquellos que asistieron a una o más sesiones de repaso.

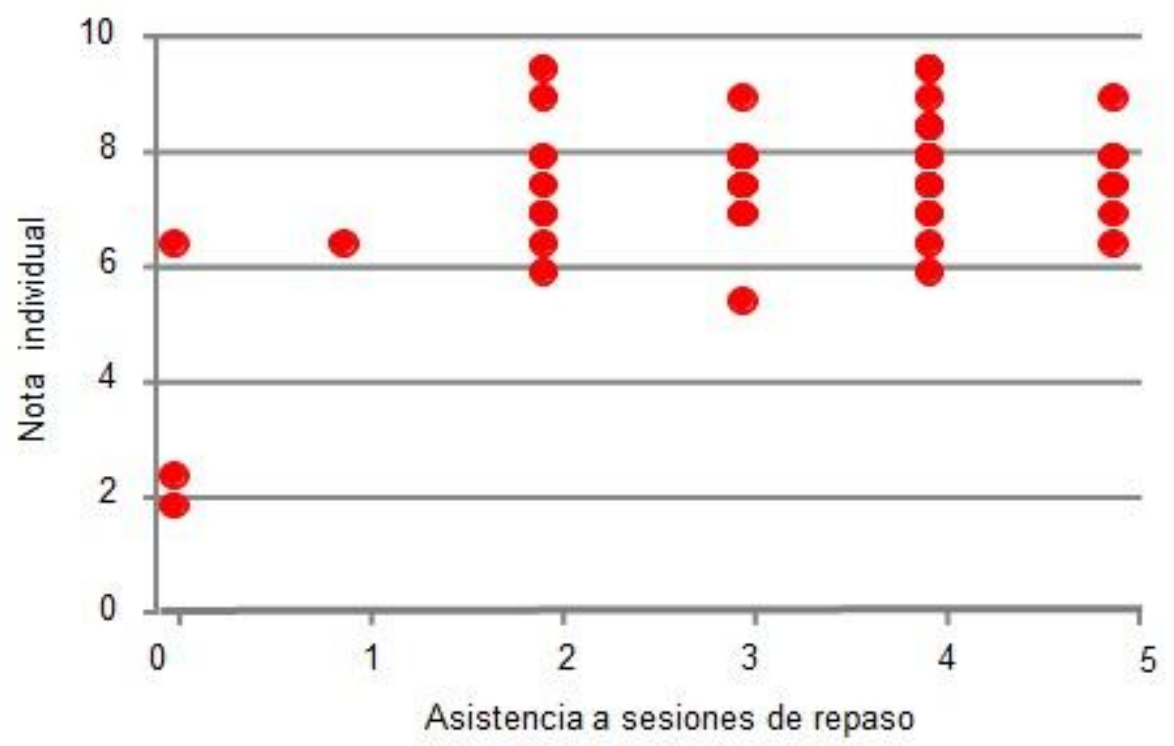

Figura 2. Notas obtenidas en el curso, según la cantidad de sesiones de repaso que asistieron los estudiantes previo al examen

\subsection{Resultados de la introducción del $25 \%$ de evaluación de actividades de aprendizaje cooperativo en el aprovechamiento y rendimiento estudiantil}

Según se observa en la Figura No.3, al hacer un análisis retrospectivo, entre el año 2006 y 2010 se obtuvieron porcentajes de aprobación del curso MQ-203 Bioquímica para Microbiología menores al $80 \%$, cuando la evaluación del aprendizaje de los estudiantes se basaba únicamente en exámenes de selección única. 
Para el año 2011 se obtuvo un porcentaje de aprobación del 94\%. Al aplicar un análisis estadístico para una media, se obtuvo un valor $p<0.05$ ( $p=0.0001$, media=71.67, $D E=14.65$ ), lo cual indica que sí hay una diferencia significativa en los porcentajes de aprobación en el curso dado en el 2011 con respecto a años anteriores. Al implementar estrategias de enseñanza cooperativa, así como la evaluación de estas, el porcentaje de estudiantes que aprobó el curso fue significativamente mayor en comparación con los años 2006 al 2010.

En otros contextos geográficos y sociales fuera de América Latina, se ha demostrado que los cursos de bioquímica que incorporan actividades de aprendizaje cooperativo conllevan a un mayor aprendizaje por parte de los estudiantes, en comparación con el aprendizaje en cursos tradicionales basados exclusivamente en actividades didácticas de carácter transmisivo centradas en el profesor (Anderson et al., 2005; Peters, 2005; Fernández-Santander, 2008; Magnarelli et al., 2009).

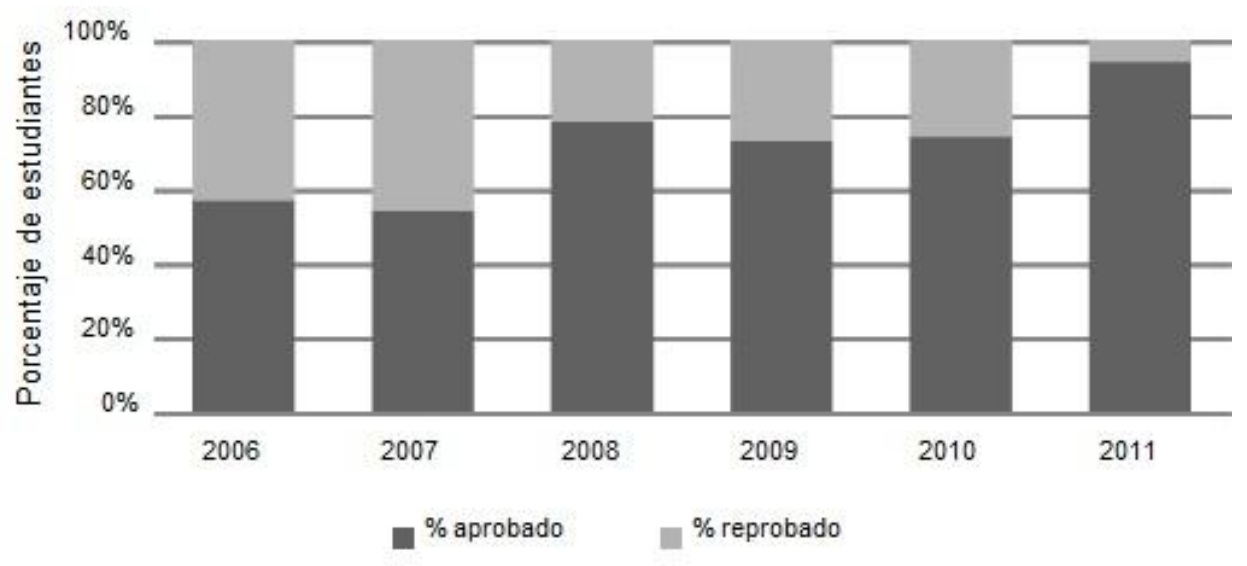

Figura 3. Porcentaje de aprobación de los estudiantes que llevaron el curso MQ-203 Bioquímica para Microbiología, del año 2006 al 2011.

En la Figura No.4 se ilustra el rendimiento de los estudiantes al final del curso, entre los años 2006 y 2011. Al graficar la distribución de las notas obtenidas (A), se observa una distribución normal de los datos, con promedios y desviaciones de: $6.4 \pm 1.6$ en el 2006, $6.3 \pm 1.6$ en el $2007,6.8 \pm 1.4$ en el $2008,6.9 \pm 1.6$ en el $2009,6.8 \pm 1.6$ en el 2010 y de $7.8 \pm 1.1$ en el 2011 (B). Al aplicar un análisis estadístico de varianza, se obtuvo un valor $p<0.05$ $(p=<0.0001)$, con lo cual se puede afirmar con un $95 \%$ de confianza que sí hay una 
diferencia significativa en las notas obtenidas por los estudiantes entre los cursos de bioquímica impartidos entre los años 2006-2010 y 2011. Mediante la prueba LSD Fisher se determina que las notas obtenidas en el año 2011 son significativamente mayores a las obtenidas en los años anteriores.
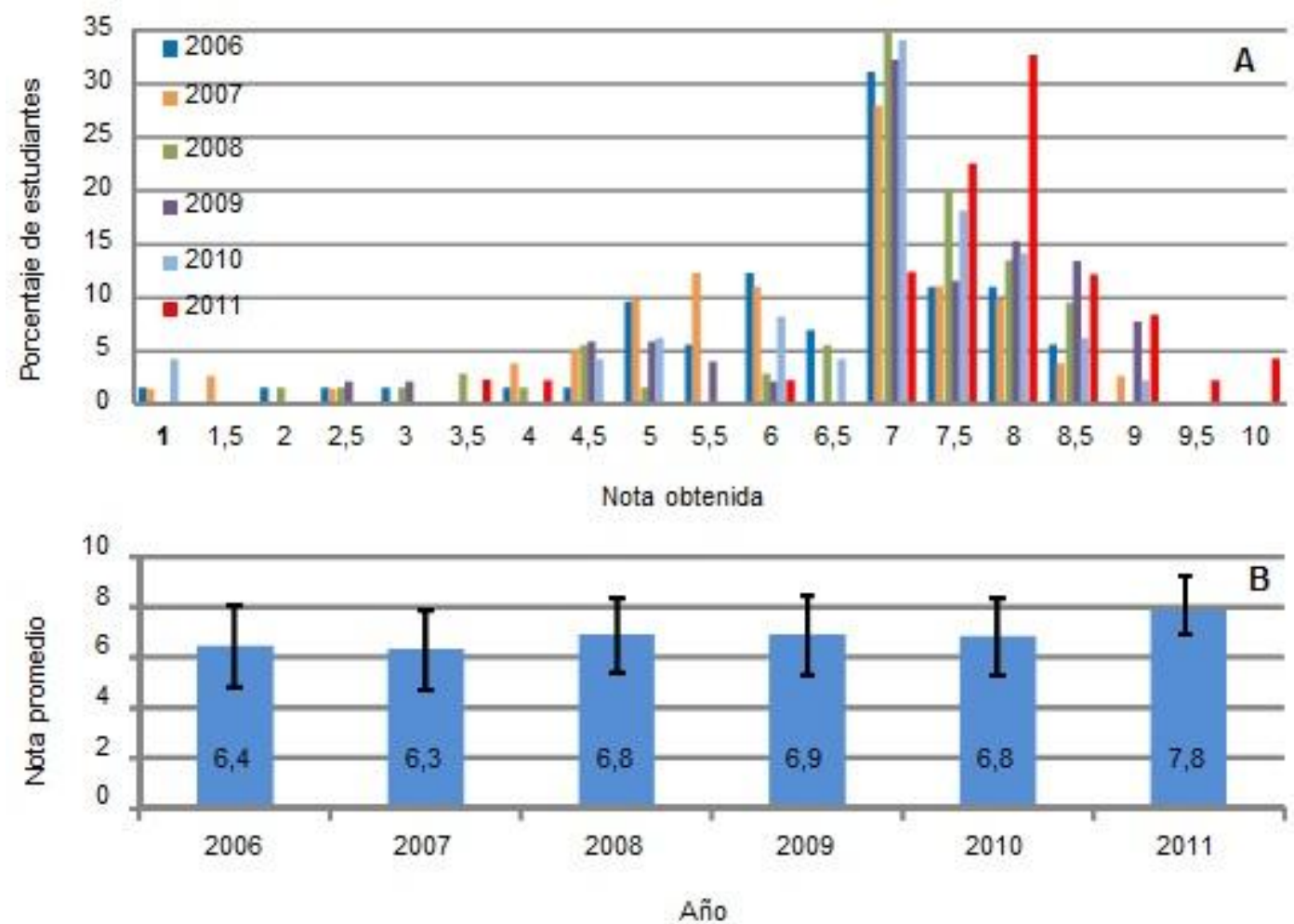

Figura 4. Rendimiento de los estudiantes del curso MQ-203

Bioquímica para Microbiología del año 2006 al 2011

Por lo tanto, la implementación de estrategias de aprendizaje cooperativo en el año 2011, y la evaluación de estas actividades como parte de la nota de los estudiantes en el curso, sí influyeron positivamente en el rendimiento de los estudiantes, al comparar con las notas obtenidas en años anteriores.

Posteriormente, se realizó una comparación de la nota final obtenida por los estudiantes en el curso, con la nota individual que hubieran obtenido de haberse omitido en 
la evaluación las actividades de aprendizaje cooperativo Según se observa en la Figura No.5, para ambas calificaciones hay una distribución normal de los datos, con una nota promedio de 7.8 \pm 1.1 para la nota final del curso (porcentaje de aprobación del 94\%), y una

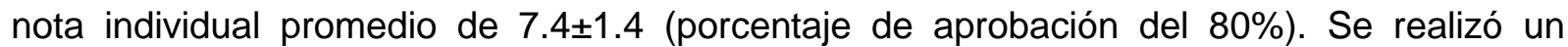
análisis de varianza para determinar si, el $25 \%$ asignado para la evaluación de las actividades en grupo favorecían o no la nota final del curso. Se obtuvo un valor $p>0.05$ $(p=0.1625)$ por lo que se puede afirmar, con un 95\% de confianza, que no hay diferencia significativa entre las notas finales obtenidas en el curso y las notas individuales. Este resultado sugiere que las buenas notas de los estudiantes se debieron, no al $25 \%$ de evaluación de las actividades en grupo, sino a que el aprendizaje cooperativo por sí sólo, mejoró el aprendizaje de los estudiantes.

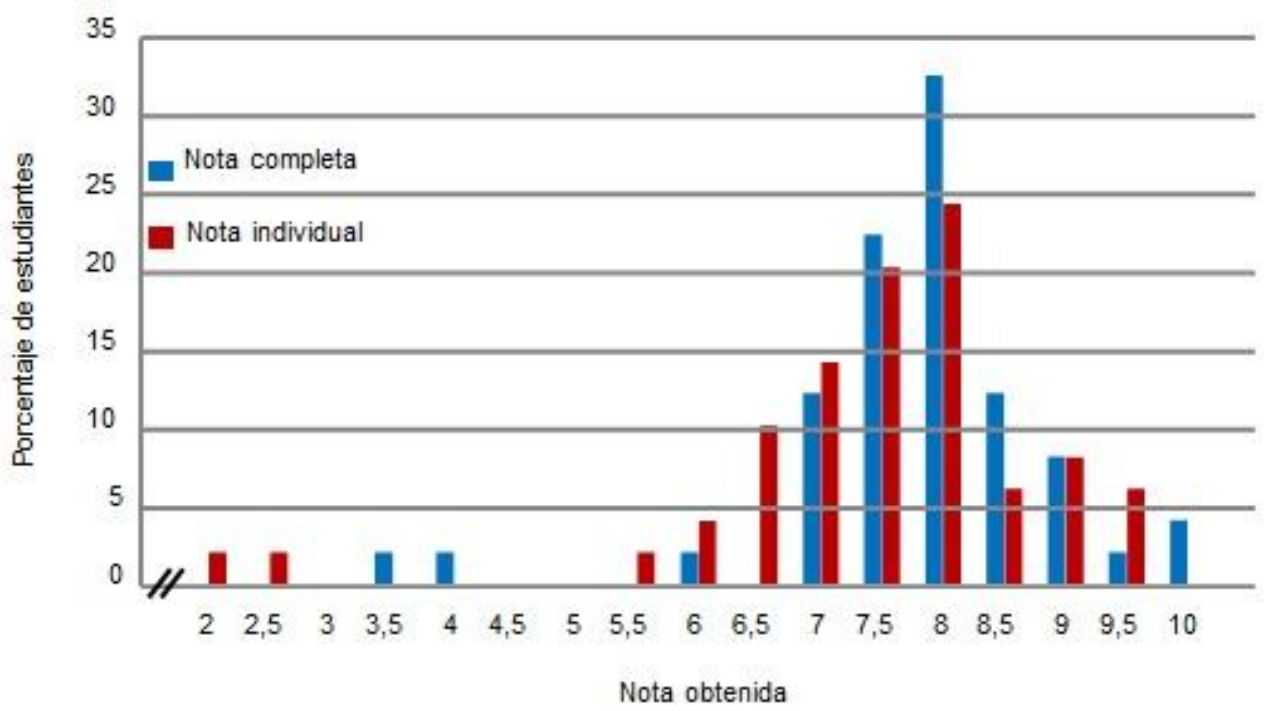

Figura 5. Rendimiento de los estudiantes del curso MQ-203 Bioquímica para Microbiología del año 2011 en base a la nota individual y nota completa.

Conociendo que, con la implementación de actividades de aprendizaje cooperativo se obtuvo una mejora en las notas de los estudiantes y el consecuente aumento en el número de estudiantes que aprobaron el curso; se procedió a comparar estos datos entre los estudiantes que llevaron el curso por primera vez (nota promedio de $8.1 \pm 1.0$ y aprobación del curso de $97.4 \%$ ), con aquellos que lo habían llevado dos o más veces (nota promedio de 
$6.9 \pm 1.3$ y aprobación del curso de $82 \%$ ). En la Figura No. 6 se observa una distribución normal de las notas.

Al realizar un análisis estadístico de varianza, se obtuvo un valor $p<0.05(p=0.0023)$, con lo que se puede afirmar, con un 95\% de confianza, que sí hay una diferencia significativa en las notas obtenidas por ambos grupos de estudiantes. Al aplicar la prueba LSD Fisher, se determinó que los estudiantes repitentes obtuvieron notas significativamente inferiores a las obtenidas por los estudiantes que llevaban el curso por primera vez. De esta manera, se determinó que los estudiantes que han llevado varias veces el curso tienen mayores dificultades para aprobarlo.

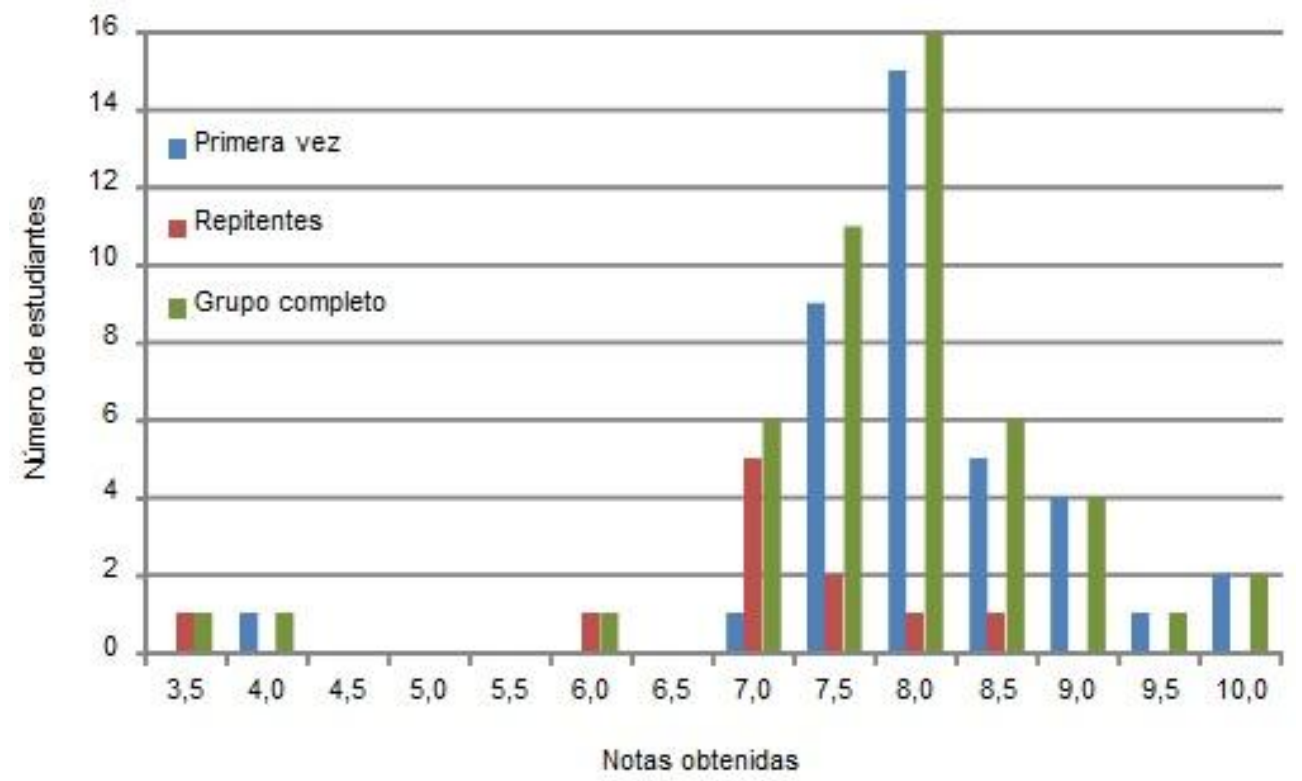

Figura 6. Rendimiento de los estudiantes del curso MQ-203 en el 2011 bajo la implementación de actividades de aprendizaje cooperativo

\subsection{Perspectiva final de los estudiantes, con respecto a la introducción de actividades cooperativas en el aprendizaje}

Al finalizar el semestre (semana 18), se les solicitó a los estudiantes contestar un cuestionario para que reflexionaran con respecto a la estructura y desarrollo del curso, así como el proceso de aprendizaje de cada uno al implementar la metodología de aprendizaje cooperativo (Cuadro 3). 


\section{CUADRO 3.}

Cuestionario evaluación final curso MQ-203 Bioquímica para Microbiología

Califique en una escala de 1 a 10 en cuánto han contribuido a su formación profesional y/o a su proceso de aprendizaje, algunos elementos o actividades del curso.

Tareas en grupo

Actividades en grupo realizadas en la clase

8.1

Los casos clínicos

9.7

El anteproyecto de investigación

9.1

El trabajo escrito del proyecto de investigación,

9.6

La presentación oral de su seminario

9.7

Las presentaciones de los seminarios de sus compañeros

9.2

Califique en una escala de 1 a 10 en cuánto la participación en este curso contribuyó para el desarrollo de los siguientes aspectos:

Capacidad de pensar de manera creativa, crítica y autocrítica

El interés en la búsqueda de mejora continua

9.4

Capacidad para trabajar de manera armónica y eficaz en grupo

9.2

Capacidad para entender las perspectivas de los otros

9.2

Habilidad para debatir respetando las opiniones ajenas y, buscando el consenso a través $\quad 9.2$

de la argumentación

Capacidad de analizar, sintetizar, relacionar e integrar información

9.2

Habilidad para integrarse socialmente con respeto, tolerancia y solidaridad

9.1

Capacidad de aprender en forma autónoma

8.7

Deseo de superación constante y búsqueda de la excelencia

9.4

Espíritu de sana competencia

8.7

Actitud científica e interés por investigar de manera sistemática las causas de los

9.4

problemas

Habilidad para comunicarse en forma clara y fluida de manera oral

8.7

Habilidad para comunicarse en forma clara y fluida de manera escrita

8.7

Interés y habilidad para buscar, analizar y valorar publicaciones científicas especializadas

9.3

Capacidad de razonar críticamente al enfrentarse a una historia clínica

9.5

Capacidad para administrar su tiempo eficientemente y llevar la materia al día

De forma global, usted calificaría este curso con una nota de:

Los comentarios finales de los estudiantes con respecto al curso y la implementación de actividades de aprendizaje cooperativo fueron muy positivos, evidenciando su satisfacción con el nuevo modelo. Entre las actividades en grupo que ellos consideraron que habían contribuido en mayor medida a su formación profesional fueron los casos clínicos, el anteproyecto de investigación, el trabajo escrito del proyecto de investigación, la presentación oral del seminario y las presentaciones de los seminarios de sus compañeros, esto debido a que eran actividades que les hizo ser autodidactas, mejorar la expresión oral y no estudiar sólo por una nota sino por aprender. Los estudiantes comentaron que la 
participación en el curso había contribuido a desarrollar el interés en la búsqueda de mejora continua, la capacidad de trabajar en grupo con respeto, tolerancia y solidaridad, logrando el consenso a través de la comunicación. Varios comentaron que el curso les había despertado el deseo de superación y búsqueda de la excelencia.

\section{Conclusiones}

Los resultados de la experiencia permiten concluir que el cambio en la metodología del curso MQ-203 Bioquímica para Microbiología mediante la implementación de actividades de aprendizaje cooperativo logró un aumento significativo en el rendimiento de los estudiantes y en los porcentajes de aprobación del curso, en comparación con años anteriores cuando este se basaba en clases magistrales.

A la vez, incrementó el interés de los alumnos por la asignatura. Los estudiantes mostraron una gran satisfacción con la nueva metodología y se involucraron más con el proceso de aprendizaje, teniendo así una mejor actitud hacia la materia. Se demostró que algunos factores socioeconómicos influyen negativamente en el rendimiento académico de los estudiantes, mientras que las interacciones sociales mejoran el desempeño en el curso, lo que promueve la construcción de nuevos conocimientos que servirán de base para el entendimiento de los cursos subsiguientes de la carrera de microbiología y el desarrollo futuro del profesional.

La metodología empleada en el curso de Bioquímica analizado, demanda una mayor participación tanto de los estudiantes como de los profesores involucrados en el proceso, es de esperar que requiera también de un mayor aporte de recurso económico para su ejecución, debido a costos de materiales y mayor recurso docente. Al considerar la implementación de este tipo de intervenciones se debe tomar en cuenta el tamaño de los grupos, la disponibilidad y disposición de los profesores, la temática a considerar en el curso y los aspectos externos que pueden contribuir a que el proceso sea exitoso. Esta experiencia nos permite recomendar su aplicación en aquellos cursos que, siendo básicos en la formación académica del estudiante, presentan bajos porcentajes de aprobación. 


\section{Agradecimientos}

Esta investigación fue realizada gracias al apoyo de la Dra. Libia Herrero, Vicerrectora de Docencia de la UCR. Se agradece la ayuda brindada por el M.Sc. Sergio Solano, la Master Arlyne Solano González, la Sra. Patricia Avendaño Mena y al Sr. Mario Andrés Barquero Campos.

\section{Referencias}

Anderson, William; Mitchell, Steven; Osgod, Marcy. (2005). Comparison of student performance in cooperative learning and traditional lecture-based biochemistry. Biochemistry and Molecular Biology Education, 33(6), 387-393.

Baghcheghi, Nayereh; Koohestani, Hamid Reza; Rezaei, Koresh. (2011). A comparison of the cooperative learning and traditional learning methods in theory classes on nursing students' communication skill with patients at clinical settings. Nurse Education Today, 31(8), 877-882.

Baumberger-Henry, Mary. (2005). Cooperative learning and case study: does the combination improve students' perception of problem-solving and decision making skills? Nurse Education Today, 25, 238-246.

Chew, Esyin; Jones, Norah; Turner, David. (2008). Critical Review of the Blended Learning Models Based on Maslow's and Vygotsky's Educational Theory. En J. Fong, R. Kwan, and F.L. Wang (Ed.). Hybrid Learning and Education (pp. 40-53). Germany: Editorial Springer-Verlag Berlin Heidelberg.

De los Ríos, Ignacio; Cazorla, Adolfo; Díaz-Puente, José M; Yagü, José L. (2010). Projectbased learning in engineering higher education: two decades of teaching competences in real environments. Procedia Social and Behavioral Sciences, 2, 1368-1378.

Domingo, Joan. (2008). El aprendizaje cooperativo. Cuadernos de Trabajo Social, 21, 231246.

Fernández-Santander, Ana. (2008). Cooperative learning combined with short periods of lecturing. Biochemistry and Molecular Biology Education, 36(1), 34-38.

Gopichandran, Vijayaprasad; Lai, Lien; Gopalan, Venkat. (2007). Protein-Energy Malnutrition. En R. Glew, and M.Rosenthal (Ed.), Clinical Studies in Medical Biochemistry (pp 255-265). England: Oxford University Press.

Hijzen, Daphne; Boekaerts, Monique; Vedder, Paul. (2007). Exploring the links between students' engagement in cooperative learning, their goal preferences and appraisals of instructional conditions in the classroom. Learning and Instruction, 17, 673-687. 
Johnson, David W., Johnson, Roger T; Smith, Karl A. (1998). Cooperative Learning Returns To College: What Evidence Is There That It Works? Change, 30(4), 27-35.

Magnarelli, Gladis; Quintana, María Martha; García, Laura; Cabrera, Luis; Ruiz, Lidia. (2009) El trabajo en pequeños grupos facilita la enseñanza-aprendizaje de Bioquímica. Revista Brasileira de Educaçao Médica, 33(3), 374-381.

Murray, Robert. (2003). Biochemistry \& Medicine. En R.K. Murray, D.K. Granner, P.A. Mayes, V.W. Rodwell (Ed.), Harper's Illustrated Biochemistry (pp. 1-4). United States: Lange Medical Books/McGraw-Hill Medical Publishing Division.

Musa, Faridah; Mufti, Norlaila; Latiff, Rozmel Abdul; Ami, Maryam Mohamed. (2011). Projectbased Learning: Promoting Meaningful Language Learning for Workplace Skills. Procedia Social and Behavioral Sciences, 18, 187-195.

Nuthall, Graham. (2000). The Role of Memory in the Acquisition and Retention of Knowledge in Science and Social Studies Units. Cognition and Instruction, 18(1), 83-139.

Peters, Angela. (2005). Teaching biochemistry at a minority-serving institution: an evaluation of the role of collaborative learning as a tool for science mastery. Journal of Chemical Education, 82(4), 571-574.

Prescott, Lansing. (2002). Preface. En Prescott, Lansing; Harley, John; Klein, Donald (Ed.), Microbiology (pp. XV-XVI). United States of America: McGraw-Hill Higher Education.

Quirolo, Keith. (2007). Sickle Cell Anemia. En Glew, and M.Rosenthal (ed.), Clinical Studies in Medical Biochemistry (pp 17-29). England: Oxford University Press.

Rybarczyk,Bryan; Baines, Antonio; McVey, Mitch; Thompson, Joseph; Wilkins, Heather. (2007). A Case-based Approach Increases Student Learning Outcomes and Comprehension of Cellular Respiration Concepts. Biochemistry and molecular biology education, 35(3), 181-186.

Sand-Jecklin, Kari. (2007). The impact of active/cooperative instruction on beginning nursing student learning strategy preference. Nurse Education Today, 27, 474-480.

Schwarzenberg, Sarah Jane; Sharp, Harvey. (2007). a1-Antitrypsin Deficiency. En R. Glew, and M.Rosenthal (Ed.), Clinical Studies in Medical Biochemistry (pp. 42-53). England: Oxford University Press.

Smagorinsky, Peter. (2007). Vygotsky and the Social Dynamics of Classrooms. English Journal, 97(2), 61-66. 
1. Licenciada en Microbiología y Química Clínica de la Universidad de Costa Rica. Profesora interina de la Escuela de Medicina de la Universidad de Costa Rica y de la Escuela de Ciencias Biológicas de la Universidad Nacional. Dirección electrónica: marialaura.fernandez@ucr.ac.cr

2. Ph.D en Bioquímica del Instituto Karolinska. Catedrático de la Escuela de Medicina de la Universidad de Costa Rica, e investigador del Instituto Clodomiro Picado y el Centro de Investigación en Estructuras Microscópicas de la Universidad de Costa Rica. Dirección electrónica: alberto.alape@ucr.ac.cr

3. M.Sc. en Bioquímica de la Universidad de Costa Rica. Profesora interina de la Escuela de Medicina de la Universidad de Costa Rica. Dirección electrónica: maria.artolozaga@ucr.ac.cr

4. Licenciado en Medicina y Cirugía de la Universidad de Costa Rica. Profesor interino de la Escuela de Medicina de la Universidad de Costa Rica.Dirección electrónica: Icalvob@yahoo.com

5. M.Sc. en Ciencias Biomédicas con énfasis en Fisiología Celular de la Universidad de Costa Rica. Profesora e Investigadora interina de la Escuela de Medicina de la Universidad de Costa Rica y del Centro de Investigación en Biotecnología del Instituto Tecnológico de Costa Rica. Dirección electrónica: carolina.centeno cerdas@ucr.ac.cr

6. M.Sc. en Ciencias Biomédicas con énfasis en Bioquímica, y M.Sc. en Nutrición de Humana de la Universidad de Costa Rica. Catedrática de la Escuela de Medicina de la Universidad de Costa Rica. Dirección electrónica: georgina.gomez@ucr.ac.cr

7. Bachiller en Biología con énfasis en Biotecnología de la Universidad Nacional. Profesora interina de la Escuela de Medicina de la Universidad de Costa Rica. Dirección electrónica: karolandrea.granados@ucr.ac.cr

8. Bachiller en Ingeniería en Biotecnología del Instituto Tecnológico de Costa Rica. Profesor interino de la Escuela de Medicina de la Universidad de Costa Rica e investigador del Instituto Clodomiro Picado. Dirección electrónica: marvin.madrigal v@ucr.ac.cr

9. Licenciada en Nutrición Humana de la Universidad de Costa Rica. Profesora interina de la Escuela de Medicina de la Universidad de Costa Rica. Dirección electrónico: anagabriela.murillo@ucr.ac.cr

10. Ph.D. en Microbiología Ambiental de la Universidad de Wisconsin. Profesor invitado de la Escuela de Medicina de la Universidad de Costa Rica e investigador del Centro de Investigación en Estructuras Microscópicas de la Universidad de Costa Rica. Dirección electrónica: adrian.pinto@ucr.ac.cr

11. Ph.D. en Biología Humana, Medizinische Hochschule Hannover, Alemania. Profesor Asociado de la Escuela de Medicina de la Universidad de Costa Rica. Dirección electrónica: silvia.quesada@ucr.ac.cr

12. Ph.D en Neurociencias con énfasis en Bioquímica y Biología Molecular de la Universidad Complutense de Madrid. Profesora invitada de la Escuela de Medicina de la Universidad de Costa Rica, e investigadora del Programa de Investigación en Neurociencias de la Universidad de Costa Rica. Dirección electrónica: elvira.salas@ucr.ac.cr

13. Bachiller en Biología con énfasis en biotecnología de la Universidad Nacional. Profesor interino de la Escuela de Medicina de la Universidad de Costa Rica. Dirección electrónica: luis.somarribaspatterson@ucr.ac.cr

14. Licenciada en Microbiología y Química Clínica de la Universidad de Costa Rica. Profesora interina de la Escuela de Medicina de la Universidad de Costa Rica. Dirección electrónica: luisavindas@gmail.com

15. Estudiante de Licenciatura de la carrera de Microbiología y Química Clínica de la Universidad de Costa Rica. Dirección electrónica: diescr@hotmail.com

Artículo recibido: 16 de setiembre, 2011

Aprobado: 16 de febrero, 2012 